\title{
Combined fibrinogen concentration and neutrophil-lymphocyte ratio as a prognostic marker of gastric cancer
}

\author{
TAKAAKI ARIGAMI $^{1,2}$, YOSHIKAZU UENOSONO ${ }^{2}$, DAISUKE MATSUSHITA ${ }^{1}$, SHIGEHIRO YANAGITA ${ }^{1}$, \\ YASUTO UCHIKADO ${ }^{1}$, YOSHIAKI KITA ${ }^{1}$, SHINICHIRO MORI ${ }^{1}$, YUKO KIJIMA ${ }^{1}$, HIROSHI OKUMURA ${ }^{1}$, \\ KOSEI MAEMURA ${ }^{1}$, SUMIYA ISHIGAMI ${ }^{1}$ and SHOJI NATSUGOE ${ }^{1,2}$ \\ Departments of ${ }^{1}$ Digestive, Breast and Thyroid Surgery and ${ }^{2}$ Molecular Frontier Surgery, \\ Kagoshima University Graduate School of Medical and Dental Sciences, Kagoshima, Kagoshima 890-8520, Japan
}

Received February 7, 2015; Accepted November 5, 2015

DOI: $10.3892 / 01.2015 .4049$

\begin{abstract}
Certain patients with early gastric cancer succumb to recurrent disease and cancer-associated complications. The key cause of recurrence is challenging to determine, since clinical blood markers that are able to predict the tumor properties of gastric cancer are limited. The present study investigated the fibrinogen concentration and neutrophil-lymphocyte ratio (NLR) in blood specimens from patients with gastric cancer, and assessed the clinical applicability of combining the fibrinogen concentration with the NLR (CFS-NLR) as a prognostic marker of gastric cancer. The present study consisted of 275 patients with gastric cancer, who were divided into three groups: Those possessing hyperfibrinogenemia ( $\geq 305 \mathrm{mg} / \mathrm{dl}$ ) and a high NLR ( $\geq 2.34$; CFS-NLR 2 group); those possessing either hyperfibrinogenemia or a high NLR (CFS-NLR 1 group); or those that possessed neither abnormality (CFS-NLR 0 group). The CFS-NLR was significantly associated with the depth of tumor invasion, lymph node metastasis, lymphovascular invasion and tumor stage $(\mathrm{P}<0.0001)$. The prognostic differences among the three groups were significant $(\mathrm{P}=0.0016)$. Therefore, the CFS-NLR may be a potentially useful blood marker for predicting tumor progression and the prognosis of patients with gastric cancer.
\end{abstract}

\section{Introduction}

Gastric cancer is the fifth most common cancer in the world, and is the third leading cause of cancer-associated mortality (1). The ability to recognize patients with early gastric cancer has

Correspondence to: Dr Takaaki Arigami, Department of Digestive, Breast and Thyroid Surgery, Kagoshima University Graduate School of Medical and Dental Sciences, 8-35-1 Sakuragaoka, Kagoshima, Kagoshima 890-8520, Japan

E-mail: arigami@m.kufm.kagoshima-u.ac.jp

Key words: fibrinogen, neutrophil-lymphocyte ratio, blood marker, prognosis, gastric cancer gradually improved due to a novel endoscopic approach that uses narrow band imaging. The prognosis of patients has also improved, with 5-year survival rates of $95-100 \%$ and $85-95 \%$ for mucosal and submucosal gastric cancer, respectively (2). However, certain patients with early gastric cancer develop and succumb to recurrent disease or cancer-associated complications. The key cause of disease recurrence is challenging to predict during the clinical management of patients with early gastric cancer. By contrast, the development of novel chemotherapy has improved the prognosis of patients with unresectable, advanced or recurrent gastric cancer (3). Although the prognosis of patients with advanced gastric cancer is poor, for patients that are recurrence-free there is a positive prognosis (3). However, predicting the postoperative prognosis of patients with advanced gastric cancer is challenging. Carcinoembryonic antigen (CEA) and carbohydrate antigen 19-9 (CA 19-9) have been used in clinical settings as conventional blood markers of gastric cancer. However, the sensitivity and specificity of immunoassays to detect these tumor markers, in order to identify subclinical patients at high risk of recurrence, is clinically limited (4).

Hepatocytes mainly synthesize fibrinogen, which is an acute-phase reactant glycoprotein (5). Activated thrombin transforms fibrinogen to fibrin in the coagulation cascade; therefore, fibrinogen is important in the regulation of clot formation, wound healing, and the inflammatory response (6). Furthermore, previous studies have demonstrated that elevated fibrinogen activity enhances tumor aggressiveness in several malignancies, including esophageal, stomach and colorectal carcinomas (7-9). Consequently, hyperfibrinogenemia is associated with tumor progression and a poor prognosis for patients with malignant disease (7-9).

The host immunosurveillance system is important in the prevention of tumor progression, and an imbalance facilitates tumor proliferation and metastasis (10). Previous studies have investigated the clinical significance of the systemic inflammatory response during tumor progression in various malignancies (11). The neutrophil-lymphocyte ratio (NLR) is a representative index that may be used to assess the systemic inflammatory response (12). Several studies have associated an elevated, preoperative NLR with tumor development and poor prognosis of patients with esophageal, hepatocellular and 
colorectal cancer, including gastric cancer (13-16). However, fibrinogen activity and the NLR have not been simultaneously assessed as markers of tumor progression and prognosis in patients with gastric cancer.

The present study aimed to determine the preoperative fibrinogen concentrations and NLRs in patients with gastric cancer, and to evaluate the association between these values and clinicopathological characteristics, including prognosis. The present study also assessed the clinical applicability of combining the fibrinogen concentration with the NLR (CFS-NLR) as a prognostic marker of gastric cancer.

\section{Materials and methods}

Patients. The present study enrolled 275 patients (179 men and 96 women; age range, 31-89 years; average age, 66 years) with gastric cancer, who underwent gastrectomy with lymphadenectomy at Kagoshima University Hospital (Kagoshima, Kagoshima, Japan) between January 2000 and December 2011. A total of 87 patients that had received endoscopic treatment, palliative resection or preoperative chemotherapy or radiotherapy were excluded. The patients were categorized and their tumors were staged based on the criteria in the tumor-node-metastasis (TNM) classification of gastric carcinoma established by the Union for International Cancer Control (17). Table I reveals the clinicopathological characteristics of the patients enrolled in the present study. All patients were followed up every 3-6 months subsequent to discharge from the hospital, by regular clinical examinations, including tumor marker studies (CEA and CA19-9), radiography, ultrasonography and computed tomography (CT) scans at Kagoshima University Hospital. The median follow-up period following surgery was 40 months (range, 1-123 months).

The Institutional Review Board of Kagoshima University Hospital approved the present study (approval no. 25-39). Prior to surgery, written informed consent was obtained from all the patients regarding the use and publication of their information.

Fibrinogen levels and NLR in blood samples. Blood specimens were pre-operatively collected to quantify the fibrinogen concentrations and NLR. Plasma fibrinogen concentrations were measured using an STA-R Coagulation Analyzer 1999 (Roche Diagnostics K.K., Tokyo, Japan). Neutrophils and lymphocytes were counted using an XE-2100 ${ }^{\mathrm{TM}}$ Automated Hematology Analyzer (Sysmex Corporation, Kobe, Hyogo, Japan). The NLR was calculated by dividing the neutrophil count by the lymphocyte count.

Scoring system of modified Glasgow prognostic score ( $m G P S$ ). The patients were divided into three groups, using cutoff values adjusted in a previous study (18), as follows: Hypoalbuminemia $(<3.8 \mathrm{~g} / \mathrm{dl})$ and a high C-reactive protein (CRP) $(>0.5 \mathrm{mg} / \mathrm{dl})$, (mGPS 2 group); either hypoalbuminemia or a high CRP, (mGPS 1 group); or neither abnormality (mGPS 0 group).

Statistical analysis. The differences in the association between fibrinogen concentrations or the NLR and categorical clinicopathological characteristics were assessed using the Wilcoxon signed-rank test. Receiver operating characteristic (ROC) curves and the area under the curve (AUC) were used to assess the
Table I. Clinicopathological characteristics of 275 patients with gastric cancer.

Characteristics n

\section{Gender}

Male 179

Female 96

Age, years

$\leq 70$

$>70$

Histological type

Differentiated

Undifferentiated

Depth of tumor invasion

pT1

pT2

pT3

pT4

Lymph node metastasis

Negative

Positive

Lymphatic invasion

Negative

Positive

Venous invasion

Negative

Positive

Tumor stage

I

II

III

IV

pT1, invasion of lamina propria or submucosa; pT2, invasion of muscularis propria; pT3, invasion of subserosa; pT4, penetration of serosa or invasion of adjacent structures.

predictive value of fibrinogen concentrations and NLR to discriminate between gastric cancer patients with advanced stages II-IV and those with early stage I. The association between CFS-NLR and the clinicopathological characteristics was analyzed using the $\chi^{2}$ test. Kaplan-Meier survival curves were generated, and the differences in survival rate were determined using the log-rank test. Prognostic factors were assessed by univariate and multivariate analyses (Cox's proportional hazards regression model). The data was analyzed using SAS/STAT ${ }^{\circledR}$ software (SAS Institute Inc., Cary, NC, USA). $\mathrm{P}<0.05$ was considered to indicate a statistically significant difference.

\section{Results}

Fibrinogen concentrations and clinicopathological characteristics. The present study initially investigated the association between clinicopathological characteristics and fibrinogen 
A

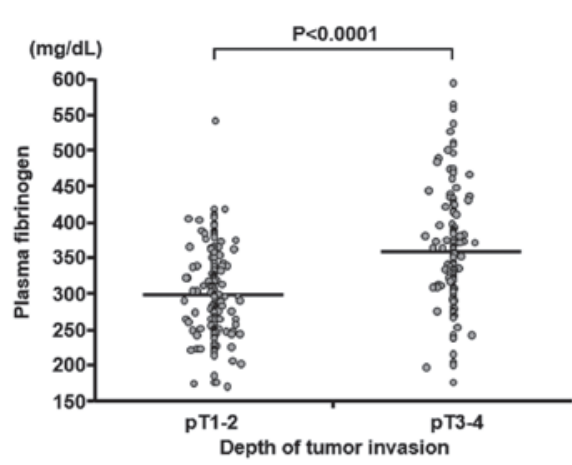

C $(\mathrm{mg} / \mathrm{dL})$

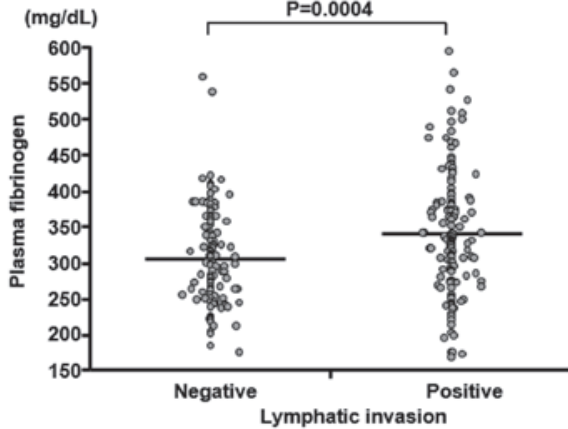

$\mathbf{E}$

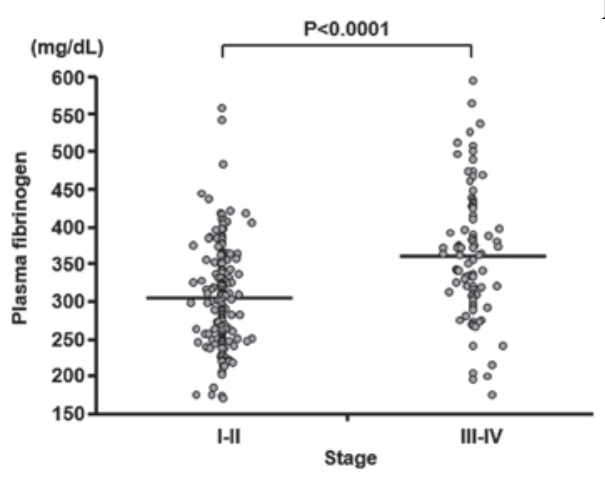

B

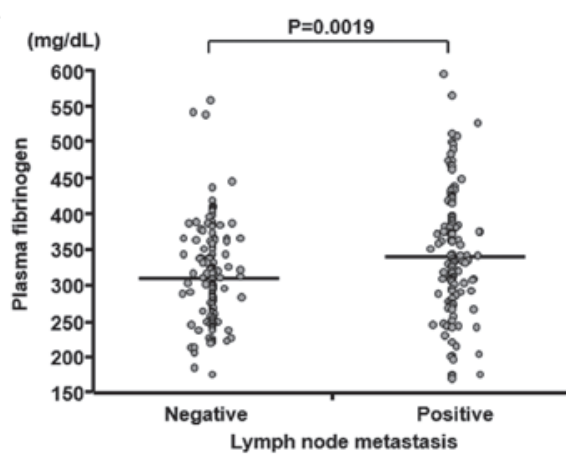

D

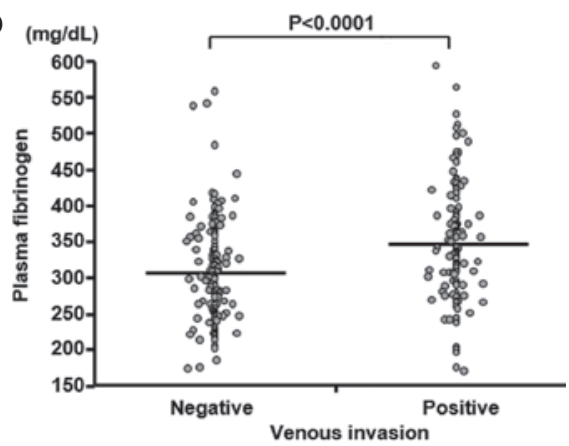

F

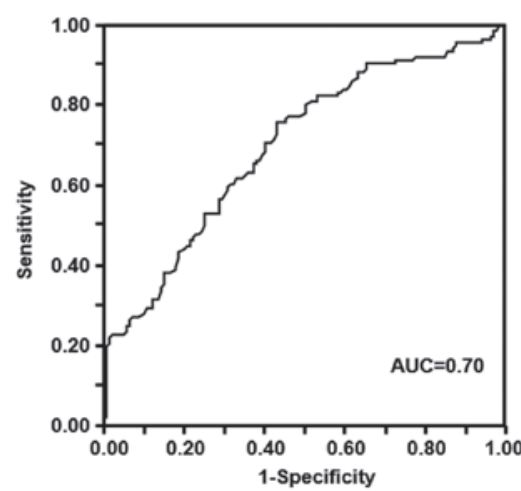

Figure 1. Association between plasma fibrinogen concentrations and clinicopathological characterisitcs in 275 patients with gastric cancer. The plasma fibrinogen concentration was significantly associated with (A) the depth of tumor invasion, (B) lymph node metastasis, (C) lymphatic invasion, (D) venous invasion and (E) tumor stage. Horizontal bars indicate the mean concentrations of plasma fibrinogen. (F) Receiver operating characteristic curve for discriminating between advanced and early gastric cancer based on plasma fibrinogen concentrations. pT1, invasion of lamina propria or submucosa; pT2, invasion of muscularis propria; pT3, invasion of subserosa; pT4, penetration of serosa or invasion of adjacent structures.

concentrations and assessed the clinical significance of the association during tumor progression in 275 patients with gastric cancer.

The mean concentration \pm standard deviation $(\mathrm{SD})$ and the range of plasma fibrinogen concentrations were $326.5 \pm 76.7 \mathrm{mg} / \mathrm{dl}$ and $171-595 \mathrm{mg} / \mathrm{dl}$, respectively. The mean $\pm \mathrm{SD}$ of the plasma fibrinogen concentrations in patients with pT1-T2 $(n=155)$ and pT3-4 $(n=120)$ tumors were $300.6 \pm 61.5$ and $360.0 \pm 81.5 \mathrm{mg} / \mathrm{dl}$, respectively (Fig. 1A). The plasma fibrinogen concentration was significantly associated with the depth of tumor invasion $(\mathrm{P}<0.0001)$. The mean $\pm \mathrm{SD}$ of the plasma fibrinogen concentrations were significantly higher in patients with lymph node metastasis $(n=130)$ than in patients without $(\mathrm{n}=145)(341.9 \pm 84.1$ and $312.8 \pm 66.8 \mathrm{mg} / \mathrm{dl}$, respectively; $\mathrm{P}=0.0019$; Fig. 1B). Furthermore, the plasma fibrinogen concentrations were associated with lymphatic and venous invasion $(\mathrm{P}=0.0004$ and $\mathrm{P}<0.0001$, respectively; Fig. $1 \mathrm{C}$ and $\mathrm{D})$. The mean $\pm \mathrm{SD}$ of the plasma fibrinogen concentrations in stage I-II $(n=183)$ and stage III-IV $(n=92)$ were $308.2 \pm 65.6$ and $362.9 \pm 84.3 \mathrm{mg} / \mathrm{dl}$, respectively (Fig. 1E). The tumor stage was also significantly associated with plasma fibrinogen concentrations $(\mathrm{P}<0.0001)$. The outcome of the ROC analysis demonstrated that the sensitivity of the AUC value for discriminating between patients with advanced and early gastric cancer based on plasma fibrinogen concentrations was 0.70 (Fig. 1F). The cutoff plasma fibrinogen concentration was $305 \mathrm{mg} / \mathrm{dl}$. The sensitivity and specificity of the plasma fibrinogen concentration was 0.76 and 0.57 , respectively. The patients were assigned to the high $(n=163)$ or low $(n=112)$ plasma fibrinogen groups based on the cutoff value. This binary system in the plasma fibrinogen level was used in subsequent analyses.

NLR and clinicopathological characteristics. The present study also assessed the association between the NLR and clinicopathological characteristics of patients during 
Table II. Association between CFS-NLR and the clinicopathological characteristics of patients with gastric cancer.

\begin{tabular}{|c|c|c|c|c|}
\hline \multirow[b]{2}{*}{ Characteristics } & \multicolumn{3}{|c|}{ CFS-NLR group, n (\%) } & \multirow[b]{2}{*}{ P-value } \\
\hline & Group 0 & Group 1 & Group 2 & \\
\hline Total & $80(100.0)$ & $130(100.0)$ & $65(100.0)$ & \\
\hline Gender & & & & 0.1960 \\
\hline Male & $52(65.0)$ & $79(60.8)$ & $48(73.9)$ & \\
\hline Female & $28(35.0)$ & $51(39.2)$ & $17(26.1)$ & \\
\hline Age, years & & & & 0.5540 \\
\hline$\leq 70$ & $50(62.5)$ & $74(56.9)$ & $35(53.8)$ & \\
\hline$>70$ & $30(37.5)$ & $56(43.1)$ & $30(46.2)$ & \\
\hline Tumor location & & & & 0.0720 \\
\hline Upper & $21(26.2)$ & $26(20.0)$ & $23(35.4)$ & \\
\hline Middle & $35(43.8)$ & $61(46.9)$ & $18(27.7)$ & \\
\hline Lower & $24(30.0)$ & $43(33.1)$ & $24(36.9)$ & \\
\hline Histological type & & & & 0.0730 \\
\hline Differentiated & $36(45.0)$ & $55(42.3)$ & $18(27.7)$ & \\
\hline Undifferentiated & $44(55.0)$ & $75(57.7)$ & $47(72.3)$ & \\
\hline Depth of tumor invasion & & & & $<0.0001$ \\
\hline pT1-T2 & $64(80.0)$ & $75(57.7)$ & $16(24.6)$ & \\
\hline pT3-T4 & $16(20.0)$ & $55(42.3)$ & $49(75.4)$ & \\
\hline Lymph node metastasis & & & & $<0.0001$ \\
\hline Negative & $55(68.8)$ & $71(54.6)$ & $19(29.2)$ & \\
\hline Positive & $25(31.2)$ & $59(45.4)$ & $46(70.8)$ & \\
\hline Lymphatic invasion & & & & $<0.0001$ \\
\hline Negative & $53(66.3)$ & $60(46.2)$ & $20(30.8)$ & \\
\hline Positive & $27(33.7)$ & $70(53.8)$ & $45(69.2)$ & \\
\hline Venous invasion & & & & $<0.0001$ \\
\hline Negative & $59(73.8)$ & $73(56.2)$ & $20(30.8)$ & \\
\hline Positive & $21(26.2)$ & $57(43.8)$ & $45(69.2)$ & \\
\hline Tumor stage & & & & $<0.0001$ \\
\hline I-II & $69(86.3)$ & $91(70.0)$ & $23(35.4)$ & \\
\hline III-IV & $11(13.7)$ & $39(30.0)$ & $42(64.6)$ & \\
\hline
\end{tabular}

CFS-NLR, combined fibrinogen concentration and neutrophil-lymphocyte ratio; pT1, invasion of lamina propria or submucosa; pT2, invasion of muscularis propria; pT3, invasion of subserosa; pT4, penetration of serosa or invasion of adjacent structures.

gastric cancer progression. In the present 275 patients, the mean $\pm \mathrm{SD}$ and range of the NLR were $2.21 \pm 1.25$ and $0.46-8.40 \mathrm{mg} / \mathrm{dl}$, respectively. The mean \pm SD of the NLR in patients with pT1-T2 $(n=155)$ and pT3-4 $(n=120)$ tumors were $1.91 \pm 0.94$ and $2.59 \pm 1.48 \mathrm{mg} / \mathrm{dl}$, respectively (Fig. 2A). This indicates a significant association between the NLR and the depth of tumor invasion $(\mathrm{P}<0.0001)$. The mean $\pm \mathrm{SD}$ of the NLR was significantly higher in patients with lymph node metastasis $(n=130)$ compared with patients without lymph node metastasis $(n=145)(2.57 \pm 1.47$ vs. $1.89 \pm 0.90 \mathrm{mg} / \mathrm{dl}$; $\mathrm{P}<0.0001$; Fig. 2B). Furthermore, the NLR was associated with lymphatic and venous invasion $(\mathrm{P}=0.0002$ and $\mathrm{P}<0.0001$, respectively; Fig. 2C and D). The mean $\pm \mathrm{SD}$ of the NLR was significantly higher in patients with stage III-IV disease $(n=92)$ compared to patients with stage I-II $(n=183)$ gastric cancer $(2.77 \pm 1.60$ vs. $1.93 \pm 0.91 \mathrm{mg} / \mathrm{dl} ; \mathrm{P}<0.0001$; Fig. $2 \mathrm{E})$. The
AUC for discriminating between patients with advanced and early gastric cancer based on the NLR was 0.63 , according to ROC analysis (Fig. 2F). Therefore, the cutoff NLR was 2.34. The sensitivity and specificity for NLR were 0.46 and 0.76 , respectively. All patients were classified into groups according to the NLR ratios, $\geq 2.34$ ( $n=97)$ or $<2.34(n=178)$. This binary system for NLR, such as for the plasma fibrinogen concentration, was used in subsequent analyses.

Fibrinogen concentration and NLR as prognostic factors. The 5-year survival rate was significantly decreased among patients with high fibrinogen values compared with those with low values (75.4 vs. $91.8 \%$; $\mathrm{P}=0.0067$; Fig. $3 \mathrm{~A}$ ) and the prognosis was significantly poorer for patients with NLR $\geq 2.34$ compared to those with NLR $<2.34$ (74.6 vs. $86.6 \%$; $\mathrm{P}=0.0206$; Fig. 3B). 
A

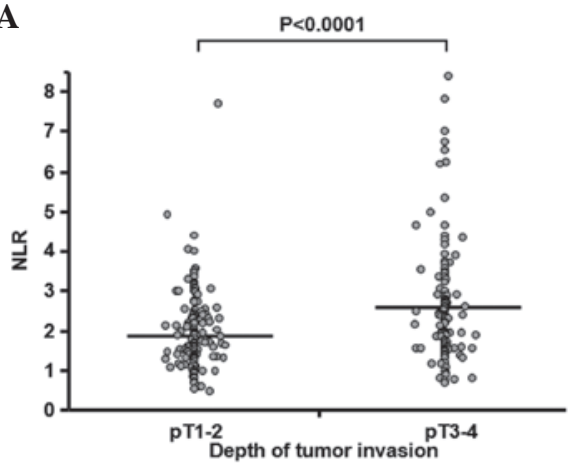

C

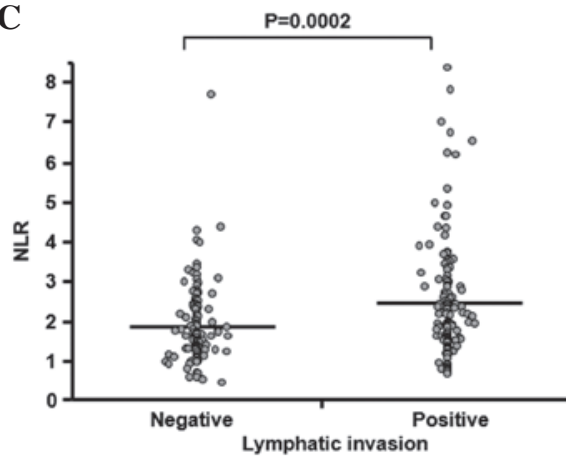

$\mathbf{E}$

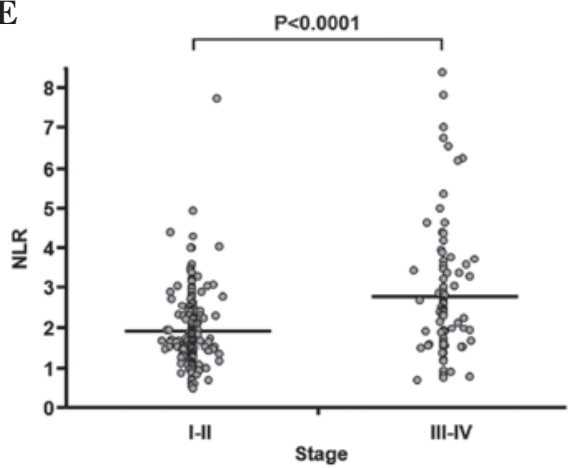

B

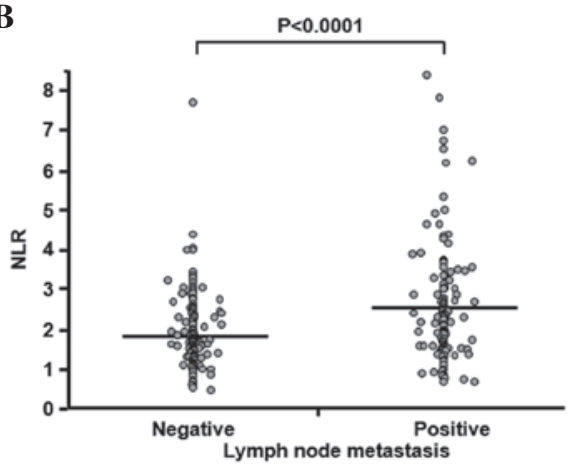

D

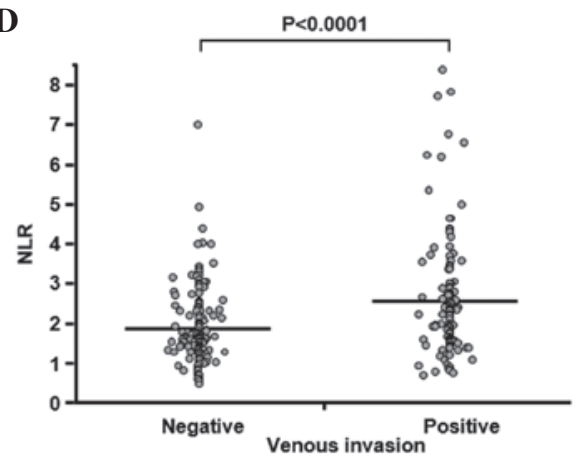

F

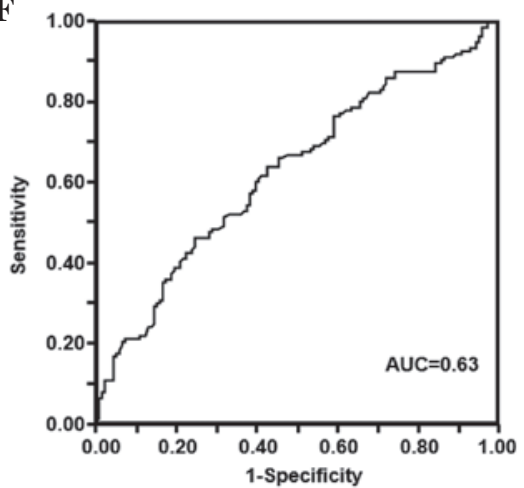

Figure 2. Association between NLR and clinicopathological characteristics in 275 patients with gastric cancer. The NLR was significantly associated with (A) the depth of tumor invasion, (B) lymph node metastasis, (C) lymphatic invasion, (D) venous invasion and (E) tumor stage. Horizontal bars indicate the mean NLR. (F) Receiver operating characteristic curve for discriminating between advanced and early gastric cancer based on NLR. NLR, neutrophil-lymphocyte ratio. $\mathrm{pT} 1$, invasion of lamina propria or submucosa; $\mathrm{pT} 2$, invasion of muscularis propria; $\mathrm{pT} 3$, invasion of subserosa; $\mathrm{pT} 4$, penetration of serosa or invasion of adjacent structures.

CFS-NLR and clinicopathological characteristics. The present study simultaneously assessed the fibrinogen concentration and NLR as CFS-NLR based on each cutoff value established in previous prognostic analyses, to determine the clinical value of CFS-NLR for patients with gastric cancer. The patients were divided into three groups, based on the following criteria: Hyperfibrinogenemia ( $\geq 305 \mathrm{mg} / \mathrm{dl}$ ) and a high NLR ( $\geq 2.34$ ); (CFS-NLR 2 group; $n=80 ; 29.1 \%$ ); either hyperfibrinogenemia or a high NLR (CFS-NLR 1 group; $\mathrm{n}=130 ; 47.3 \%$ ); or neither abnormality (CFS-NLR 0 group; $\mathrm{n}=65 ; 23.6 \%$ ).

The CFS-NLR was significantly associated with the depth of tumor invasion, lymph node metastasis, lymphovascular invasion and tumor stage $(\mathrm{P}<0.0001$; Table II).

CFS-NLR as a prognostic factor. The 5-year survival rates of patients in CFS-NLR groups 0,1 and 2 differed significantly and were $92.9,84.1$ and $66.5 \%$, respectively $(\mathrm{P}=0.0016$; Fig. 4$)$.
Univariate and multivariate analyses of the survival rate. The depth of tumor invasion, lymph node metastasis and CFS-NLR were significantly associated with prognosis in the univariate analysis $(\mathrm{P}<0.0001, \mathrm{P}<0.0001$ and $\mathrm{P}=0.0018$, respectively; Table III). Multivariate analysis demonstrated that the depth of tumor invasion and lymph node metastasis were independent prognostic factors $(\mathrm{P}=0.01$ and $\mathrm{P}<0.0001$, respectively; Table III).

$m G P S$ as a prognostic factor. The 5 year survival rates of patients in mGPS groups $0(n=183 ; 66.5 \%), 1(n=64 ; 23.3 \%)$ and $2(\mathrm{n}=28 ; 10.2 \%)$ differed significantly and were $87.1,75.0$ and $63.9 \%$, respectively $(\mathrm{P}=0.0055)$.

\section{Discussion}

The present study analyzed plasma fibrinogen concentrations and the NLR in pre-operative blood specimens obtained from 275 patients with gastric cancer. The fibrinogen concentrations 
Table III. Univariate and multivariate analyses of the survival rate of patients with gastric cancer.

\begin{tabular}{|c|c|c|c|c|c|c|}
\hline \multirow{2}{*}{$\begin{array}{l}\text { Independent } \\
\text { prognostic factor }\end{array}$} & \multicolumn{3}{|c|}{ Univariate analysis } & \multicolumn{3}{|c|}{ Multivariate analysis } \\
\hline & Hazard ratio & $95 \%$ CI & P-value & Hazard ratio & $95 \% \mathrm{CI}$ & P-value \\
\hline \multicolumn{7}{|c|}{ Depth of tumor invasion } \\
\hline $\mathrm{pT} 1-\mathrm{T} 3 / \mathrm{pT} 4$ & 4.59 & $2.37-8.87$ & $<0.0001$ & 2.44 & $1.24-4.82$ & 0.0100 \\
\hline \multicolumn{7}{|c|}{ Lymph node metastasis } \\
\hline Negative/Positive & 12.77 & $4.58-53.10$ & $<0.0001$ & 8.80 & $3.04-37.27$ & $<0.0001$ \\
\hline \multicolumn{7}{|l|}{ CFS-NLR } \\
\hline Group $0-1 / 2$ & 2.94 & $1.51-5.67$ & 0.0018 & 1.87 & $0.95-3.64$ & 0.0695 \\
\hline
\end{tabular}

CI, confidence interval; pT1, invasion of lamina propria or submucosa; pT3, invasion of subserosa; pT4, penetration of serosa or invasion of adjacent structures; CFS-NLR, combined fibrinogen concentration and the neutrophil-lymphocyte ratio.
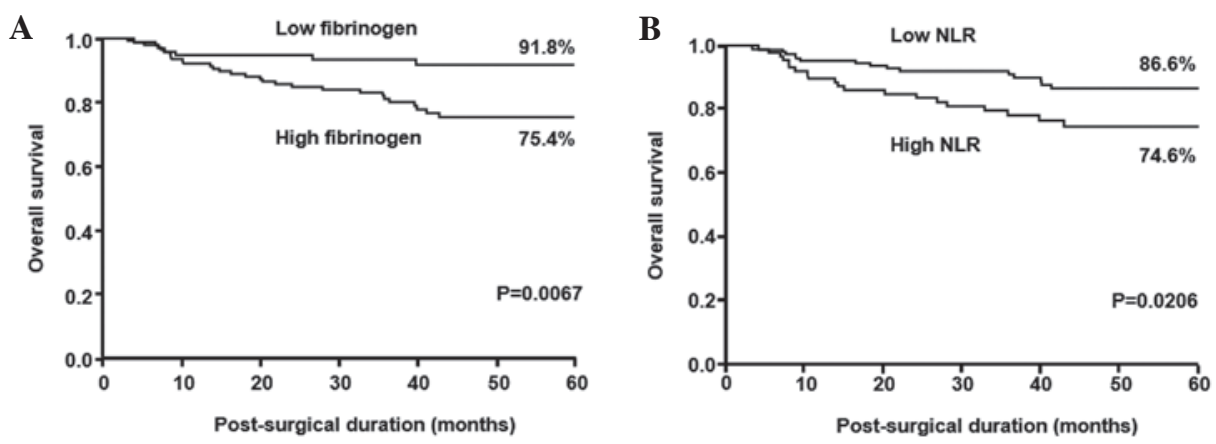

Figure 3. Kaplan-Meier survival curves for patients with gastric cancer based on (A) plasma fibrinogen concentrations and (B) NLRs. NLR, neutrophil-lymphocyte ratio.

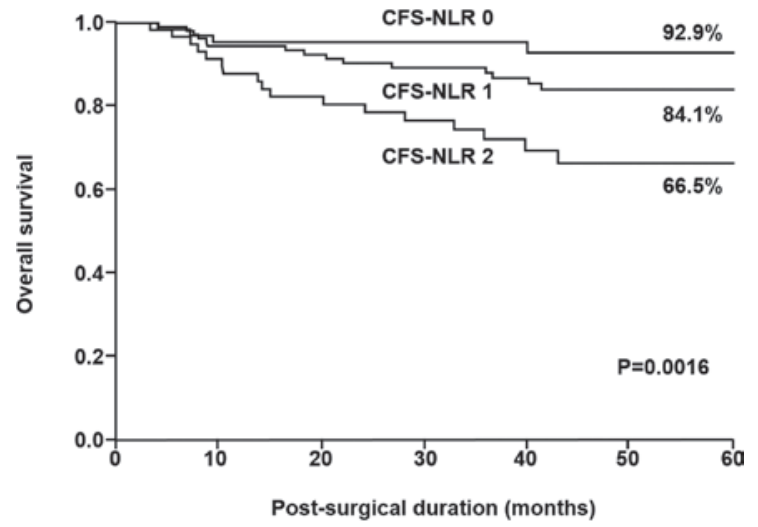

Figure 4. Kaplan-Meier survival curves for patients with gastric cancer based on CFS-NLR. CFS-NLR, combined fibrinogen concentration and the neutrophil-lymphocyte ratio.

and NLR were assessed together as the CFS-NLR and its association with clinicopathological characteristics, including prognosis, was investigated. To the best of our knowledge, this is the first study to determine the clinical value of CFS-NLR in patients with gastric cancer.

Several studies have associated hyperfibrinogenemia with tumor progression and the development of metastasis in patients with various malignancies (7-9); however, a reliable basis for elucidating this mechanism remains unknown. Yamaguchi et al (19) reported that interleukin-6, which is produced by cancer cells, stimulates fibrinogen secretion in patients with lung cancer. Furthermore, cancer cells synthesize fibrinogen (20). Fibrinogen eventually promotes tumor cell growth and angiogenesis through the interaction with fibroblast growth factor-2 and vascular endothelial growth factor $(20,21)$. The present study identified a significant association between hyperfibrinogenemia and known clinicopathological prognostic characteristics, including the depth of tumor invasion, lymph node metastasis, lymphovascular invasion and tumor stage. Furthermore, the findings that 5-year survival rates were significantly decreased in patients with increased fibrinogen concentrations indicated that evaluating plasma fibrinogen may be useful for the preoperative prediction of progressive gastric cancer and prognosis.

Inflammatory cells in primary and metastatic tumor foci are important in the process of tumor cell growth, and tumor cells create an advantageous microenvironment by suppressing antitumor immunity, which enables their progression (22). A previous study identified an improved prognosis when patients with gastric cancer had a high, rather than a low, density of cluster of differentiation (CD) $3^{+}$tumor-infiltrating lymphocytes (23). Zhao et al (24) revealed that gastric cancer patients with a higher density of $\mathrm{CD} 5^{+}$tumor-infiltrating neutrophils possessed a poorer prognosis. A systemic inflammatory response 
causes variations in the balance of circulating white blood cell constituents (25). These findings indicate that the systemic inflammatory response mediates increases and decreases in the numbers of neutrophils and lymphocytes, respectively, during cancer progression. The NLR has been previously investigated as a representative marker indicating a systemic inflammatory response, in patients with various malignant neoplasms (13-16). The present study demonstrated that the NLR is closely associated with the depth of tumor invasion, lymph node metastasis, lymphovascular invasion and tumor stage. Furthermore, the 5-year survival rate was significantly decreased for patients with a higher NLR. These findings suggest that the NLR is a potential blood marker for predicting tumor aggressiveness, in connection with the host microenvironment. Certain studies have previously revealed that the NLR may be a useful predictor of chemosensitivity in patients with advanced cancer $(26,27)$.

The most salient feature of the present study was the assessment of the plasma fibrinogen concentration and NLR as a novel index, CFS-NLR. The present study identified a close association between CFS-NLR and the depth of tumor invasion, lymph node metastasis and lymphovascular invasion, which are prominent clinicopathological prognostic characteristics in patients with gastric cancer. However, to diagnose these characteristics in practice using preoperative imaging modalities, including endoscopy, endoscopic ultrasonography, CT and positron emission tomography, may be challenging (28). The evaluation of CFS-NLR in preoperative blood specimens would be clinically useful for indicating the pathological status of tumor progression in combination with diagnostic imaging. Furthermore, this potential marker may aid the selection of patients with latent gastric cancer that may be candidates for neoadjuvant systemic chemotherapy. The CFS-NLR is easily calculated from fibrinogen concentrations and the NLR, which are determined by conventional blood analyses. Consequently, CFS-NLR may serve as a practical, cost-effective blood marker that does not require dedicated measuring devices.

The GPS, which is a combined index consisting of C-reactive protein (CRP) and albumin, is a prominent marker that is associated with the systemic inflammatory response during tumor progression (29). Numerous studies have demonstrated that GPS is a useful indicator of tumor aggressive behavior and prognosis in patients with various malignant neoplasms, including gastric cancer $(29,30)$. Furthermore, recent studies have proposed that the mGPS, which consists of adjusted values of CRP and albumin, is clinically useful $(18,31)$. In total, 1 study of 295 patients with gastric cancer identified a significantly poorer prognosis among patients with a higher mGPS, in terms of survival rates (18). The present study assessed the prognostic significance of mGPS in 275 patients with gastric cancer, using cutoff values adjusted in the previous study (18), to yield 3 groups. The log-rank test revealed a significant prognostic difference between the three groups with mGPS scores of 0,1 or $2(\mathrm{P}=0.0055)$. The prognostic difference between the same three groups classified by the CFS-NLR also differed significantly $(\mathrm{P}=0.0016)$. Furthermore, the CFS-NLR was significantly associated with the prognosis in a univariate analysis $(\mathrm{P}=0.0018)$. These results suggest that CFS-NLR is a more effective prognostic marker than mGPS for patients with gastric cancer. However, multivariate analysis did not select CFS-NLR as an independent prognostic factor $(\mathrm{P}=0.0695)$.
Since this result may have been affected by a short follow-up period and a small sample size, larger validation studies of patients with gastric cancer are required. Additional studies are being prepared to survey blood specimens from patients with other gastrointestinal tract cancers to assess the clinical significance of CFS-NLR.

In conclusion, the present study proposes a novel prognostic factor, the CFS-NLR, which is a combination of the fibrinogen concentration and NLR of blood specimens obtained from patients with gastric cancer. The present study demonstrates that the CFS-NLR is closely associated with tumor progression and prognosis in gastric cancer patients; therefore, CFS-NLR may act as a convenient marker to predict tumor properties.

\section{Acknowledgements}

This study was supported in part by a grant-in-aid (grant no. 22791256) for scientific research from the Ministry of Education, Science, Sports and Culture, Japan.

\section{References}

1. Fock KM: Review article: The epidemiology and prevention of gastric cancer. Aliment Pharmacol Ther 40: 250-260, 2014.

2. Maehara Y, Orita H, Okuyama T, Moriguchi S, Tsujitani S, Korenaga D and Sugimachi K: Predictors of lymph node metastasis in early gastric cancer. Br J Surg 79: 245-247, 1992.

3. Ohtsu A: Current status and future prospects of chemotherapy for metastatic gastric cancer: A review. Gastric Cancer 8: 95-102, 2005

4. Gaspar MJ, Arribas I, Coca MC and Diez-Alonso M: Prognostic value of carcinoembryonic antigen, CA 19-9 and CA 72-4 in gastric carcinoma. Tumour Biol 22: 318-322, 2001.

5. Tennent GA, Brennan SO, Stangou AJ, O’Grady J, Hawkins PN and Pepys MB: Human plasma fibrinogen is synthesized in the liver. Blood 109: 1971-1974, 2007.

6. Weisel JW: Fibrinogen and fibrin. Adv Protein Chem 70: 247-299, 2005

7. Yamashita H, Kitayama J, Kanno N, Yatomi Y and Nagawa H: Hyperfibrinogenemia is associated with lymphatic as well as hematogenous metastasis and worse clinical outcome in T2 gastric cancer. BMC Cancer 6: 147, 2006.

8. Takeuchi H, Ikeuchi S, Kitagawa Y, Shimada A, Oishi T, Isobe Y, Kubochi K, Kitajima M and Matsumoto S: Pretreatment plasma fibrinogen level correlates with tumor progression and metastasis in patients with squamous cell carcinoma of the esophagus. J Gastroenterol Hepatol 22: 2222-2227, 2007.

9. Son HJ, Park JW, Chang HJ, Kim DY, Kim BC, Kim SY, Park SC, Choi HS and Oh JH: Preoperative plasma hyperfibrinogenemia is predictive of poor prognosis in patients with nonmetastatic colon cancer. Ann Surg Oncol 20: 2908-2913, 2013.

10. Vesely MD and Schreiber RD: Cancer immunoediting: Antigens, mechanisms, and implications to cancer immunotherapy. Ann NY Acad Sci 1284: 1-5, 2013.

11. Mantovani A, Allavena P, Sica A and Balkwill F: Cancer-related inflammation. Nature 454: 436-444, 2008.

12. Guthrie GJ, Charles KA, Roxburgh CS, Horgan PG, McMillan DC and Clarke SJ: The systemic inflammation-based neutrophil-lymphocyte ratio: Experience in patients with cancer. Crit Rev Oncol Hematol 88: 218-230, 2013.

13. Shimada H, Takiguchi N, Kainuma O, Soda H, Ikeda A, Cho A, Miyazaki A, Gunji H, Yamamoto H and Nagata M: High preoperative neutrophil-lymphocyte ratio predicts poor survival in patients with gastric cancer. Gastric Cancer 13: 170-176, 2010.

14. Sharaiha RZ, Halazun KJ, Mirza F, Port JL, Lee PC, Neugut AI, Altorki NK and Abrams JA: Elevated preoperative neutrophil:lymphocyte ratio as a predictor of postoperative disease recurrence in esophageal cancer. Ann Surg Oncol 18: 3362-3369, 2011.

15. Mano Y, Shirabe K, Yamashita Y, Harimoto N, Tsujita E, Takeishi K, Aishima S, Ikegami T, Yoshizumi T, Yamanaka T and Maehara Y: Preoperative neutrophil-to-lymphocyte ratio is a predictor of survival after hepatectomy for hepatocellular carcinoma: A retrospective analysis. Ann Surg 258: 301-305, 2013. 
16. Li MX, Liu XM, Zhang XF, Zhang JF, Wang WL, Zhu Y, Dong J, Cheng JW, Liu ZW, Ma L and Lv Y: Prognostic role of neutrophil-to-lymphocyte ratio in colorectal cancer: A systematic review and meta-analysis. Int J Cancer 134: 2403-2413, 2014.

17. Edge S, Byrd DR, Compton CC, Fritz AG, Greene FL and Trotti A (eds): American Joint Committee on Cancer (AJCC) Cancer Staging Manual. 7th edition. Springer, New York, NY, pp 117-121, 2010.

18. Hirashima K, Watanabe M, Shigaki H, Imamura Y, Ida S, Iwatsuki M, Ishimoto T, Iwagami S, Baba Y and Baba $H$ : Prognostic significance of the modified Glasgow prognostic score in elderly patients with gastric cancer. J Gastroenterol 49: 1040-1046, 2014.

19. Yamaguchi T, Yamamoto Y, Yokota S, Nakagawa M, Ito M and Ogura T: Involvement of interleukin- 6 in the elevation of plasma fibrinogen levels in lung cancer patients. Jpn J Clin Oncol 28: 740-744, 1998

20. Sahni A, Simpson-Haidaris PJ, Sahni SK, Vaday GG and Francis CW: Fibrinogen synthesized by cancer cells augments the proliferative effect of fibroblast growth factor-2 (FGF-2) J Thromb Haemost 6: 176-183, 2008.

21. Sahni A and Francis CW: Vascular endothelial growth factor binds to fibrinogen and fibrin and stimulates endothelial cell proliferation. Blood 96: 3772-3778, 2000.

22. Fridman WH, Galon J, Dieu-Nosjean MC, Cremer I, Fisson S, Damotte D, Pagès F, Tartour E and Sautès-Fridman C: Immune infiltration in human cancer: prognostic significance and disease control. Curr Top Microbiol Immunol 344: 1-24, 2011.

23. Arigami T, Uenosono Y, Ishigami S, Matsushita D, Hirahara T, Yanagita S, Okumura H, Uchikado Y, Nakajo A, Kijima Y and Natsugoe S: Decreased density of CD3+ tumor-infiltrating lymphocytes during gastric cancer progression. J Gastroenterol Hepatol 29: 1435-1441, 2014

24. Zhao JJ, Pan K, Wang W, Chen JG, Wu YH, Lv L, Li JJ, Chen YB, Wang DD, Pan QZ, et al: The prognostic value of tumor-infiltrating neutrophils in gastric adenocarcinoma after resection. PLoS One 7: e33655, 2012.
25. Satomi A, Murakami S, Ishida K, Mastuki M, Hashimoto T and Sonoda M: Significance of increased neutrophils in patients with advanced colorectal cancer. Acta Oncol 34: 69-73, 1995.

26. Sato H, Tsubosa Y and Kawano T: Correlation between the pretherapeutic neutrophil to lymphocyte ratio and the pathologic response to neoadjuvant chemotherapy in patients with advanced esophageal cancer. World J Surg 36: 617-622, 2012.

27. Lorente D, Mateo J, Templeton AJ, Zafeiriou Z, Bianchini D, Ferraldeschi R, Bahl A, Shen L, Su Z, Sartor O and de Bono JS: Baseline neutrophil-lymphocyte ratio (NLR) is associated with survival and response to treatment with second-line chemotherapy for advanced prostate cancer independent of baseline steroid use. Ann Oncol 26: 750-755, 2015.

28. Sim SH, Kim YJ, Oh DY, Lee SH, Kim DW, Kang WJ, Im SA, Kim TY, Kim WH, Heo DS and Bang YJ: The role of PET/CT in detection of gastric cancer recurrence. BMC Cancer 9: 73, 2009.

29. McMillan DC: The systemic inflammation-based Glasgow Prognostic Score: A decade of experience in patients with cancer. Cancer Treat Rev 39: 534-540, 2013.

30. Vashist YK, Loos J, Dedow J, Tachezy M, Uzunoglu G, Kutup A, Yekebas EF and Izbicki JR: Glasgow Prognostic Score is a predictor of perioperative and long-term outcome in patients with only surgically treated esophageal cancer. Ann Surg Oncol 18: 1130-1138, 2011

31. Lamb GW, Aitchison M, Ramsey S, Housley SL and McMillan DC: Clinical utility of the Glasgow Prognostic Score in patients undergoing curative nephrectomy for renal clear cell cancer: Basis of new prognostic scoring systems. Br J Cancer 106: 279-283, 2012 